\title{
The positive impact of e-mail referrals on access to specialist macula services
}

\author{
Christopher King ${ }^{1} \cdot$ John Logan $\mathbb{D}^{2} \cdot$ Nicole $\operatorname{Lim}^{2} \cdot$ Colm Andrews $^{1} \cdot$ Susan M. Downes ${ }^{1,3}$
}

Received: 16 August 2018 / Accepted: 5 September 2018 / Published online: 19 October 2018

(c) The Royal College of Ophthalmologists 2018

Patients suspected of having neovascular age-related macular degeneration (nAMD) requires rapid access to specialist clinics for confirmatory diagnosis and subsequent treatment. The Royal College of Ophthalmologists (RCOphth) guidelines [1] recommend $<7$ days delay between initial referral in primary care to assessment with a specialist in macular disease, and for those patients requiring treatment $<7$ days delay between specialist consultation and initial treatment. In order to achieve this the Oxford Eye Hospital utilises the 'Wet AMD rapid access referral form' developed by the Thames Valley Macular Group, adopted by the RCOphth and endorsed by the Macular Society and RNIB for all referrals to the dedicated rapid-access clinic for patients with suspected nAMD [2]. To address the concern that rapid-access referrals were being missed and booked into routine clinics, a streamlined e-mail referral system was created using a dedicated nhs.net email account from August 2014.

Despite this system being widely shared with primary care providers. many referrals to our service are via other routes. We have audited the delay in access to specialist clinic for each referral route for 98 new AMD referrals seen in clinic between January and March 2018. Only 33.7\% of referrals were received thorough the dedicated e-mail address, with $19.4 \%$ via the walk-in eye casualty service or other sub-specialist clinics and the remaining $46.9 \%$ via the patients' general preactitioners (GPs). Figure 1 shows

Susan M. Downes

susan.downes@ouh.nhs.uk

1 Oxford Eye Hospital, John Radcliffe Hospital, Oxford University Hospitals NHS Foundation Trust, Headley Way,

Oxford OX39DU, UK

2 Oxford University Medical School, Medical Sciences Divisional Office, University of Oxford, John Radcliffe Hospital, Oxford OX39DU, UK

3 Nuffield Laboratory of Ophthalmology, Nuffield Department of Clinical Neurosciences, John Radcliffe Hospital, Headley Way, Oxford OX39DU, UK the delay between referral and first specialist review for patients in each of the three referral groups. No patients referred via their (GP) were seen within the 7 days target. The differences in the proportions of patients seen between the e-mail referral group and GP referral group within 2 weeks $(p=0.0014)$ and within 3 weeks $(p<0.0001)$ were statistically significant (chi-squared test).

Overall $28.6 \%$ of patients referred were found to have nAMD in at least one eye. Figure 2 shows the delay between referral and specialist review for those patients found to have nAMD and those with other diagnoses. It shows that many patients who are subsequently confirmed to have nAMD are facing delays in accessing specialist review.

This work shows that, while there are other challenges in seeing patients in a timely manner, the use of a dedicated email referral system for nAMD can significantly reduce delays in patients being seen in specialist macular clinics compared to traditional GP-centred referral systems, and the importance of reinforcing the use of this system by optometrists and other providers of primary ophthalmic services in the community. It also highlights the need for more training of community optometrists to ensure that the rapid access pathway is used only for nAMD.

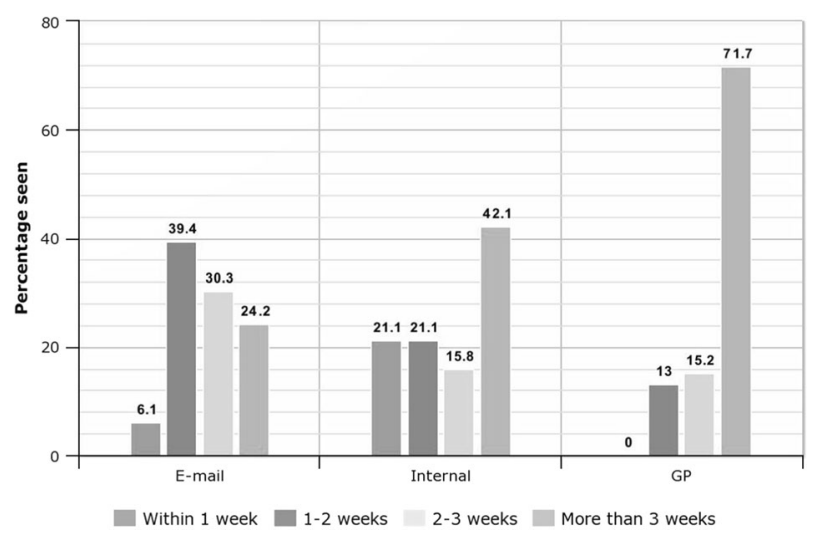

Fig. 1 Delay between referral and specialist review by referral type 


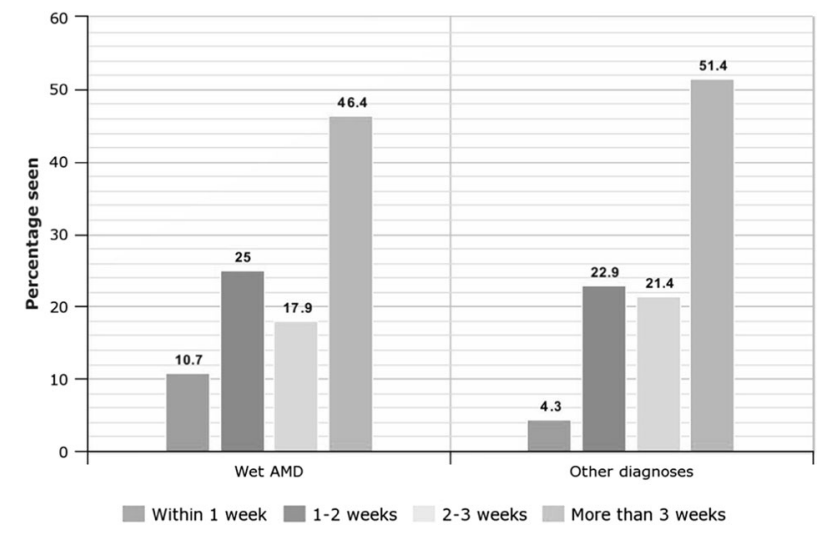

Fig. 2 Delay between referral and specialist review by diagnosis

Acknowledgements We thank the Thames Valley Macular Group Rapid Access Guidelines developed by SM Downes, C Moorman, SL Watson and L Jenkins in collaboration with the Oxfordshire LOC.

\section{Compliance with ethical standards}

Conflict of interest Susan Downes Disclosure: PI on relevant commercially sponsored trials in last 5 years: Novartis, Bayer, and
Alcon. Prof Susan Downes has received honoraria in the past 15 years from Novartis and Bayer for speaking at educational meetings, as well as travel expenses from Ely Lilly as chair for diabetic retinopathy screening meetings, and Novartis to attend educational meetings in Medical Retina pre 2010. She is a PI on a number of commercial trials (Novartis, Roche, Bayer, Allergen, among others), and has received a bursary for a research nurse practitioner, and equipment (visudyne pump for photodynamic therapy) and funding to carry out genetic testing for a research project from Novartis. She also has has been a co-PI or Pl on grants from Wellcome, UK Fighting Blindness RP, Fight for Sight and UK NIHR/CRN funding. The remaining authors declare that they have no conflict of interest.

\section{References}

1. The Royal College of Ophthalmologists. Age-Related Macular Degeneration: Guidelines for Management 2013. https://www. rcophth.ac.uk/wp-content/uploads/2014/12/2013-SCI-318RCOphth-AMD-Guidelines-Sept-2013-FINAL-2.pdf

2. The Royal College of Ophthalmologists. Wet AMD Rapid Access Referral Form 2010. https://www.rcophth.ac.uk/wp-content/uploa ds/2015/04/2010-SCI-048-AMD-Electronic-Referral-Form-edited. pdf

\title{
Accuracy of pinhole visual acuity at an urban Indian hospital
}

\author{
Rajesh S. Kumar ${ }^{1,2} \cdot$ Mahalakshmi V. Rackenchath ${ }^{1} \cdot$ A. V. Sathidevi ${ }^{1} \cdot$ Sriharsha Nagaraj $^{1} \cdot$ Caitlin A. Moe $^{3}$. \\ Robert L. Stamper ${ }^{4}$ Jeremy D. Keenan $\mathbb{1 D}^{3,4}$
}

Received: 16 August 2018 / Accepted: 7 September 2018 / Published online: 19 October 2018

(c) The Royal College of Ophthalmologists 2018

The World Health Organization-endorsed rapid assessment of avoidable blindness (RAAB) survey employs pinhole acuity to distinguish between refractive error versus conditions not correctable with eyeglasses, but few studies have validated this approach [1].

Jeremy D. Keenan

jeremy.keenan@ucsf.edu

1 Narayana Nethralaya Eye Hospital, Bangalore, India

2 Cleveland Clinic Abu Dhabi, Abu Dhabi, UAE

3 Francis I Proctor Foundation, University of California, San Francisco, CA, USA

4 Department of Ophthalmology, University of California, San Francisco, CA, USA

\section{Methods}

Ethical committees at the University of California, San Francisco and Narayana Nethralya Eye Hospital approved this study. A consecutive series of patients aged $\geq 50$ years visiting the refraction clinic at Narayana Nethralaya Eye Hospital (Bangalore, India) in September 2015 had presenting vision and pinhole vision assessed using an ETDRS chart in a fully illuminated room, and then had a manifest refraction by an experienced optometrist. Analyses are reported with bootstrapped $95 \%$ confidence intervals with resampling at the participant level to account for nonindependence of eyes. 